\title{
Surgical Excision of a Large Popliteal Varicosity in a Patient with Type 3 Von Willebrand Disease
}

\author{
Dr.Prashant Tubachi ${ }^{1}$ Dr.Annappa Kudva ${ }^{2}$ Dr.Sourabh Agarwal ${ }^{3}$ \\ Dr.Vineet Mannan ${ }^{4}$ \\ (Department of Surgery Kasturba Medical College Manipal)
}

\begin{abstract}
Von Willibrands disease is the most common inherited bleeding disorder and hepatitis $C$ a highly infective disease. Here by, we present an interesting case of a large bleeding popliteal varicosity in a patient with type 3 von Willibrands disease and hepatitis $C$ positive status. A 33 year old female patient with a bleeding popliteal varicosity was managed by pressure bandages and foot end elevation at a rural hospital. She was then referred to our center. After resuscitation, she was administered with dried human coagulation factor VIII and cryoprecipitate. On clinical examination there was a bilobed swelling in the popliteal region along with a breach in the skin. Once the patient was hemodynamically stable, Colour Doppler was done which showed a large popliteal varicosity with thrombosis and multiple perforator incompetence in the great and short saphenous territory. Bleeding parameters were optimized by human coagulation factor VIII followed by excision of the large popliteal varicosity under general anesthesia. The outcome of the treatment was remarkable.
\end{abstract}

Key Words: von Willibrand disease, varicosity bleeding.

\section{Introduction}

Lower limb varicose veins of the Great saphenous and short saphenous veins are a common entity in clinical practice and, bleeding from these varicosities is a known complication. Management of active bleeding in such varicosities is done by pressure bandage and elevation of the limb followed by definitive treatment. Whereas, in case of a large bleeding varicosity in a patient with von Willebrands type 3 disease requires management of bleeding diathesis and hemostasis by surgical excision.

\section{Case Report}

A 33 year old female patient with type 3 von Willebrands disease and hepatitis $\mathrm{C}$ having a history of bleeding from a large right popliteal varicosity was managed by pressure bandage and foot end elevation in a rural hospital and then referred to our institute. After arrival she was resuscitated in the emergency triage. As there were no signs of active bleed on bandage, dressing was not opened on the same day. Dressing was opened only after evaluating coagulation parameters and arranging for adequate blood, human coagulation factor VIII and cryoprecipitate. On examination there was a bilobed swelling in the right popliteal fossa with a breach in the skin. (Fig 1) Varicose veins involving great saphenous vein territory were also present. Colour Doppler showed GSV insufficiency with a large popliteal varicosity with thrombosis.

Past history revealed that the patient was diagnosed with von Willebrand's disease at the age of 4 years. Patient also presented with the history of bleeding from the gums every day in the morning subsiding as the day progress. She also complained of dilated veins in the lower limb since 6 years and was advised surgery for varicose veins 2 years back but she had refused for any treatment.

In menstrual history she presented with a 10 day cycle with increased bleeding for which tranexemic acid had been prescribed. There was no information about hereditary diseases or a tendency to haemorrhage among immediate family members.

Investigations revealed low $\mathrm{Hb}(8.4 \mathrm{gm} / \mathrm{dl})$, total $\mathrm{WBC}$ count of $14000 / \mu 1$, prolonged bleeding $(6 \mathrm{~min})$ and clotting time (11min), lower levels of van Willebrands factor (49.2\%) and lower fibrinogen levels (1.706 $\mathrm{g} / \mathrm{l})$. A haematologist and anaesthesiologist were consulted preoperatively. Pre and postoperatively, human coagulation factor VIII and cryoprecipitate were transfused due to the simultaneous lack of fibrinogen and type 3 von Willebrand disease. After adequate preoperative preparation, the corrected haemostasis parameters were within the normal range. Then the patient underwent surgical excision of the large popliteal varicosity (Fig 2 and 3). The operative course was uncomplicated. On the first and second postoperative days, the fibrinogen values were $1.910 \mathrm{~g} / \mathrm{l}, 2.214 \mathrm{~g} / \mathrm{l}$ and $2.880 \mathrm{~g} / \mathrm{l}$ respectively. The bleeding time measurements and haematological parameters remained within the normal range. The patient was then discharged from the hospital on the $7^{\text {th }}$ postoperative day and was prescribed to use elastic compression stockings (23-32mmHg). Small blowouts were managed with sclerotherapy on outpatient basis. 


\section{Discussion}

The von Willebrand disease (VWD) is the most common inherited bleeding disorder, with an estimated prevalence of $1 \%$ in the general population. ${ }^{[1]}$ This haemorrhagic disorder is caused by deficiency or dysfunction of the protein Von Willebrands factor. Consequently defective Von Willebrands factor interaction between platelets and vessel wall impairs primary hemostasis.

Von Willebrands disease has three types, type 1 - partial quantitative deficiency, type 2- qualitative deficiency and type 3 -total quantitative deficiency. ${ }^{[2]}$ Type 1 has been reported to be the most frequent form of Von Willebrands disease. Patients with mild form of the disorder may be asymptomatic. Whereas, severe form of the disorder presents with mucosal petechaie, easy bruising, spontaneous bleeding from the mucosa, menorrhagia or intense perioperative bleeding. ${ }^{[3]}$ Treatment depends on the severity of the disease. The different treatment options include desmopressin, cryoprecipitate and human coagulation factor VIII.

Varicose vein in the lower limb is defined as dilated subcutaneous vein $\geq 3 \mathrm{~mm}$ in diameter. ${ }^{[4]}$ Most of the varicose veins are primary venous diseases. Though etiology can be multifactorial, the origin of venous reflux in primary varicose veins can be local or multifocal structural weakness of the vein wall and that can occur together or independently of proximal saphenous incompetence. ${ }^{[5]}$ Varicosities can be seen in the great or short saphenous vein territory or any subcutaneous vein in the lower limb. Patients with varicose veins may present with no symptoms at all. Varices are a cosmetic concern only, with psychological impact. In clinical practice varicose vein patients present usually with chronic venous insufficiency. Symptoms of varicose veins or chronic venous insufficiency might be itching, edema, heaviness in legs and tiredness. ${ }^{[6]}$ Chronic venous insufficiency is the outcome of long standing varicose veins where skin changes like edema and lipodermatosclerosis are seen. Venous ulcer is another complication of chronic venous insufficiency. Venous Doppler is the investigation of choice where Saphenofemoral, saphenopopliteal valve incompetence can be identified along with perforator incompetence and deep vein patency.

There are different options to treat varicose veins. Endovenous laser and radiofrequency ablation are the preferred treatment modalities compared to surgery, as there is reduced post op pain and hospital stay ${ }^{[7]}$ and can also be used safely in patients with VonWillebrands disease. ${ }^{[8]}$ Surgery is the preferred choice of treatment in cases of large bleeding varicosities. But in cases of bleeding varicosities with associated VonWillebrands disease, optimization of the patient for surgery is a challenge. With wide availability of human coagulation factor VIII, some studies recommend administration of factor VIII concentrates as prophylaxis in patients undergoing surgery. ${ }^{[9]}$

The management of a bleeding lower limb varicosity is done by elevation of the limb to control the bleeding, followed by pressure bandage at the site. Once the bleeding is controlled definitive therapy should be carried out. If the diameter of the bleeding lower limb varicosity is less than $1 \mathrm{~mm}$ then injection sclerotherapy is tried and for a varicosity of more than $1 \mathrm{~mm}$ surgical excision is the treatment. ${ }^{[10]}$

\section{Conclusion}

In case of a bleeding varicosity in the lower limb, the definitive therapy is surgical excision once patient is stabilized. Similarly bleeding of a varicosity in a patient with von Willebrands disease is managed by transfusion of human recombinant factor VIII then surgical excision.

\section{Referances}

1. Rodeghiero F, Castaman G, Dini E. Epidemiological investigation of the prevelance of von Willebrand's disease. Blood 6,: 1987; 454-459

2. Castaman G, Montgomery RR, Meschengieser SS, Haberichter SLet a,1 Willebrand's disease diagnosis and laboratory issues. Haemophilia 16(5), 2010; 67-734

3. Zdziarska J, Chojnowki K, Klukowska A, Łętowska M et al on behalf of The Working Group of Polish Society of Haematology and Blood Transfusion Medicine. The management of von Willebrand disease. Med Praktyczna 12, 2008; 1-24

4. Kistner RL.and Eklof B, classification and etiology of chronic venous disease.in: P Glovicek (ED) handbook of venous disorders:guidelines of american venous forum $3^{\text {rd }}$ edn, Hodder Arold $2009: 37-46$

5. Labropolous N, Giannoulas AD, Delis K, Mansour MA, Kang SS, et al. Where does venous reflux start? J Vasc Surg 26, 1997,736742

6. Langer RD, Ho E, Denenberg JO, Fronek, Allison M et al. Relationships between symptoms and venous disease: the San Diego population study. Arch.Intern Med 165, 2005 1420-1424

7. Geier B, Stücker M, Hummel T, Burger P et al. Residual stumps associated with inguinal varicose vein recurrences: A multicenter study. Eur J Vasc Endovasc Surg. 36, 2008, 207-210

8. Piotr Terlecki, Tomasz Zubilewicz, Stanisław Przywara, Marek Iłżecki, Wacław Karakuła et al.Minimally invasive, endovenous laser treatment of varicose veins in patients with von Willebrand disease. Annals of agricultural and environmental medicine 20(4), $2013880-83$

9. Lethagen S, Kyrle P, CastamanG, Haertel S, et al.HAEMATE P Surgical group. Von Willebrand factor/ factor VIII concentrate dosing based on pharmacokinetics; a prospective multicenter trial in elective surgery. J Thomb Haemost 5, 2007, 1420-1430

10. Management of sudden profuse bleeding from varicose veins. McCarthy WJ1, Dann C, Pearce WH, Yao JS. Surgery 113(2), 1993, 178-83 


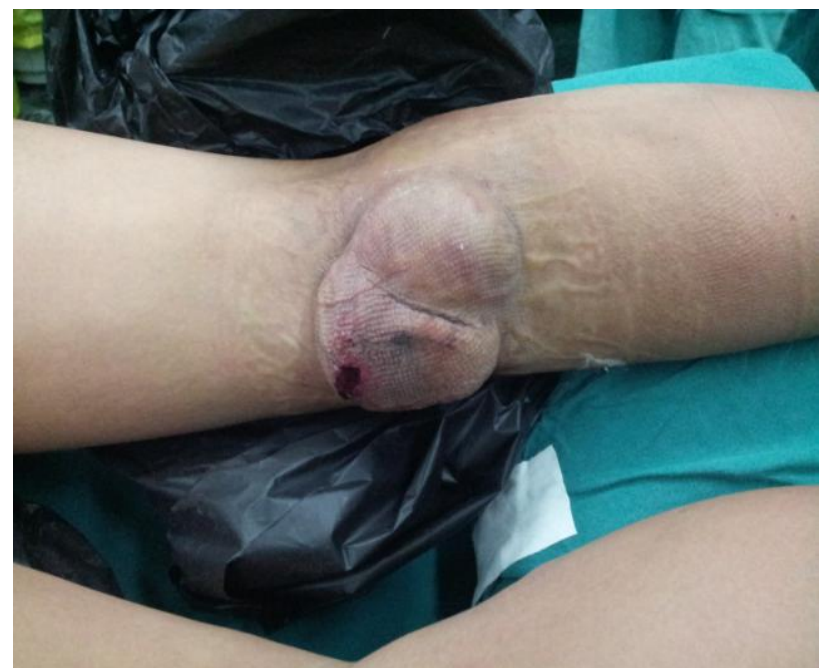

Figure 1- Photograph Showing Bilobed Varicosity Over Right Popliteal Region

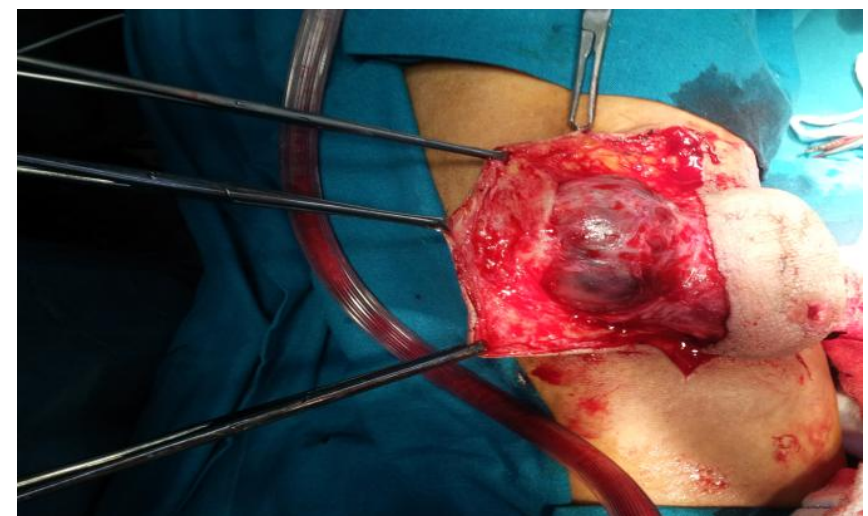

Figure 2- Intra-Op Photograph Showing The Varicosity

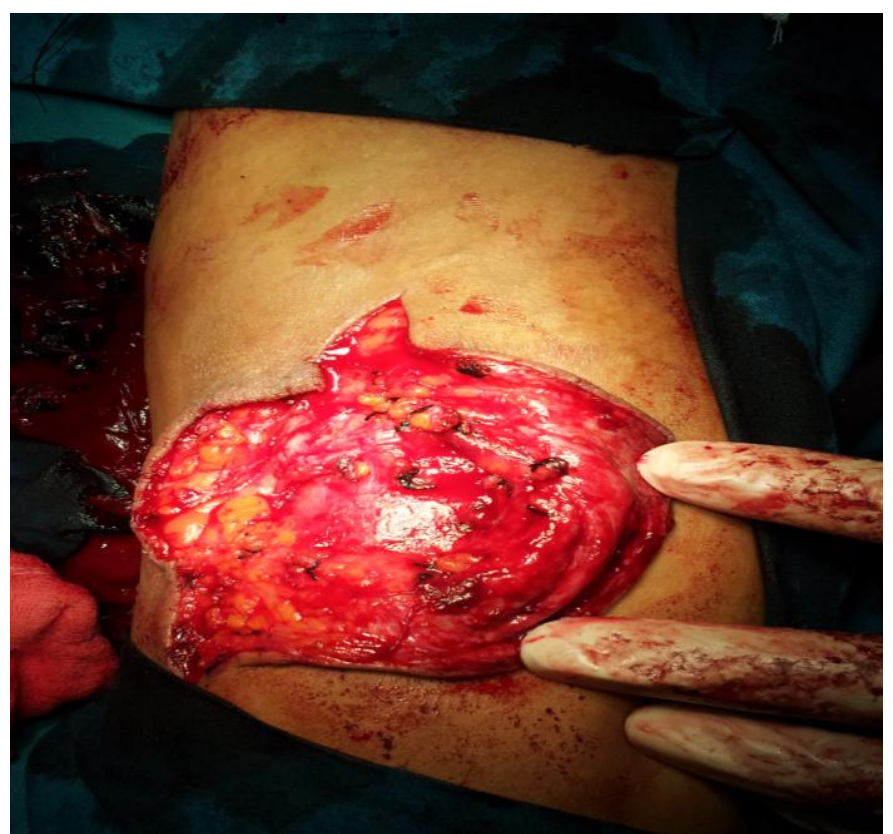

Figure 3- Intra-Op Pictire Of Defect After Complete Excision Of Varices 